Convergences francophones $2.2(2015): 25-32$

http://mrujs.mtroyal.ca/index.php/cf/index

\title{
Les préfaces des traducteurs des Anciens Canadiens: transformation du message
}

\author{
Alexandra Hillinger \\ Université Concordia, Montréal
}

De la Conquête jusqu'à la fin du XIX ${ }^{\mathrm{e}}$ siècle, la littérature écrite au Québec a pour but de servir la nation tout en s'adressant à un lectorat canadien-français. ${ }^{1}$ En 1839, Lord Durham, qui perçoit les Canadiens-français comme un peuple sans histoire et sans littérature, dépose son fameux rapport prônant leur assimilation. À partir de ce moment, le lien entre survie et production littéraire va de soi. Dans ce sens, le $\mathrm{XIX}^{\mathrm{e}}$ siècle est caractérisé par le développement de la vie littéraire dans le territoire habité par les anciens colons français. On assiste à la naissance de nombreux journaux qui contribueront à la diffusion de la littérature canadiennefrançaise.

En 1860, l'abbé Casgrain parle même d'un « mouvement littéraire en Canada ». Il va toutefois plus loin en l'investissant d'une mission : " elle sera essentiellement croyante et religieuse » (Casgrain cité Biron et al. 58). En 1874, Edmond Lareau note: "Le roman, en Canada, porte un caractère tout particulier, il est essentiellement national. Il a beaucoup contribué à donner à notre littérature son originalité, si tant est qu'elle en a une »(274). Ce caractère particulier est celui d'une production littéraire tournée vers le passé et qui vante la vie rurale et les bonnes mœurs (Linteau 329). Dans ce courant, nous retrouvons Les Anciens Canadiens (1863) de Philippe Aubert de Gaspé.

Publiée en 1863, l'œuvre d'Aubert de Gaspé est considérée comme le premier succès commercial du Canada français. Ce roman porte sur les mœurs des anciens Canadiens. Nous mentionnons ici que l'appellation « anciens Canadiens » désigne les habitants de la Nouvelle-France qui ont vécu la guerre de Sept Ans et la prise du Canada par les forces britanniques. Centré sur le passé, à savoir les années qui ont précédé et suivi la Conquête, l'ouvrage peut être qualifié de roman historique. Le but de ce roman est de documenter et de glorifier la vie et les exploits des gens qui ont vécu à cette époque. Ainsi sont retranscrites, dans l'ouvrage, la légende de la Corriveau et de nombreuses chansons populaires, éléments qui témoignent bien du thème principal du roman, la mémoire. Aubert de Gaspé couche donc sur papier les mœurs, les coutumes et les croyances d'une époque révolue.

L'œuvre d'Aubert de Gaspé a été traduite à trois reprises au cours de l'histoire. Du point de vue des traductions, Les Anciens Canadiens représente un cas de figure unique pour deux raisons. Premièrement, ce roman est traduit très rapidement, à savoir l'année suivant sa publication. D'après nos recherches, il s'agit du seul roman du XIX ${ }^{\mathrm{e}}$ siècle à faire l'objet d'une version anglaise au cours de ce

\footnotetext{
${ }^{1}$ En 1840, à la suite de l'Acte d'Union, les British Americans (les Loyalistes) prennent l'habitude de se désigner comme Canadians. Puis, pour la presse anglophone, les Canadiens de descendance française (qui jusque-là se désignaient en tant que Canadiens) deviennent des French Canadians, alors que les Loyalistes demeurent des Canadians. Ce sont donc les anglophones qui utilisent le terme Canadien-français en premier (Arès 11). Ici, nous utiliserons le terme « Canadien-Français », sans égard pour l'époque, afin d'éviter toute forme de confusion.
} 
Convergences francophones $2.2(2015): 25-32$

http://mrujs.mtroyal.ca/index.php/cf/index

même siècle. Deuxièmement, Les Anciens Canadiens a été traduit à deux reprises au XIX ${ }^{\mathrm{e}}$ siècle.

La première traduction, signée Georgiana M. Pennée, est publiée en 1864. La traduction paraît un an seulement après la sortie du roman, par contre elle ne contient pas de préface. Nous ne savons donc pas pourquoi Pennée a entrepris de rendre l'œuvre en anglais ni pourquoi la maison d'édition G. \& G.E Desparats l'a imprimée. Néanmoins, nous pouvons supposer que le succès de l'édition originale française a influencé la décision de traduire l'œuvre. Bref, l'engouement pour le roman de Gaspé aurait eu des échos jusque chez les anglophones, ce qui aurait mené à la production d'une traduction.

Les deux traductions suivantes sont accompagnées de préfaces signées par le traducteur. Dans les pages qui suivent, nous nous proposons donc d'examiner en profondeur ces écrits afin de comparer leurs messages. À notre avis, les préfaces permettent d'analyser un aspect bien particulier du contexte de production des traductions, à savoir le message que veut véhiculer le traducteur. Ainsi, il est important de garder en tête le fait que les préfaces ne contiennent que les informations que le traducteur a choisi de coucher sur papier. La préface est ce que le traducteur veut bien nous raconter à propos de son entreprise de traduction, et qu'il pourrait, volontairement ou involontairement, écarter certains détails.

La première retraduction du roman d'Aubert de Gaspé paraît en 1890. Cette traduction publiée sous le titre de Canadians of Old est signée Charles G. D. Roberts. Roberts, reconnu comme le père de la littérature canadienne, est poète, historien et nationaliste canadien. Bien qu'il ait été avare d'informations concernant son expérience de traduction, sa correspondance révèle qu'il a trouvé la tâche particulièrement ardue (Boone 120-3). Canadians of Old sera d'ailleurs sa seule incursion dans la traduction. Il faut comprendre que c'est la nécessité financière plutôt que l'intérêt pour l'œuvre de De Gaspé, ou pour la traduction en générale, qui pousse Roberts à accepter de traduire le roman de plus de 400 pages en moins d'un été (Boone 121). En effet, il ne peut pas refuser les quelque deux cents dollars que lui offre la maison d'édition Appleton.

Pour accompagner sa traduction, Roberts signe une introduction de 4 pages. D'entrée de jeu, mentionnons que la traduction Canadians of Old est publiée aux États-Unis. Ainsi, Roberts s'adresse probablement en premier lieu au lecteur de la Nouvelle-Angleterre qui connaît peu le Canada et encore moins son penchant francophone. Par contre, il se veut très fédérateur dans ses propos, et l'on sent qu'il garde en tête ses compatriotes canadiens-anglais, notamment lorsqu'il écrit «We of English speech ».

Dans ce contexte, Roberts amorce son texte en abordant le thème des « deux solitudes ». Il insiste sur le fait que le Canada compte deux nations, deux langues et deux peuples distincts. Ainsi sont produites deux littératures : « In Canada there is settling into shape of a nation of two races; there is springing into existence, at the same time, a literature in two languages » (De Gaspé 1890: 3). Roberts se garde bien d'affirmer la supériorité d'une des deux langues cultures, mais il souligne le fait que leurs existences dans un même territoire crée une série de défis. À son avis, ces défis devront être résolus à l'avenir. Bien qu'il ne propose pas de solutions pour rapprocher les deux peuples, il affirme qu'ils doivent apprendre à se connaître et à se comprendre. 
Convergences francophones $2.2(2015): 25-32$

http://mrujs.mtroyal.ca/index.php/cf/index

Ce survol de la situation entre les deux groupes linguistiques lui permet d'amener le fait que les anglophones se tournent naturellement vers la production littéraire canadienne-française pour obtenir de l'information à propos de ce peuple. Roberts inscrit sa traduction dans ce mouvement. L'œuvre d'Aubert de Gaspé, bien qu'elle ait été écrite dans le but premier de divertir le lecteur, regorge d'informations pertinentes. Il s'agit d'une juste reconstruction de la vie des premiers Canadiens-français. À la lecture de la traduction, le lecteur anglais pourra dégager les motifs et les ambitions de ce peuple (3).

Après avoir expliqué l'intérêt de l'œuvre pour le lecteur, Roberts s'affaire à en vanter les mérites. Selon lui, Les Anciens Canadiens est sans aucun doute un classique, la meilleure romance historique provenant du Canada français (3). Le roman d'Aubert de Gaspé couche sur papier, pour la pérennité, les chansons, légendes, coutumes, idées et états d'esprit d'une civilisation qui change rapidement. Bref, pour Roberts, même s'il pond sa traduction trente ans après la parution de l'original, tout l'intérêt du roman repose sur son caractère historique. La traduction permet d'offrir aux anglophones un portrait des Canadiens-français à l'époque de la Conquête.

Fait notable, Roberts se porte ensuite à la défense du nationalisme québécois. Pour lui, une personne de l'extérieur gagnerait à se familiariser avec les Canadiens-français par le biais du portrait fidèle qu'offre Canadians of Old avant de qualifier le nationalisme québécois de rêve inatteignable. En connaissant mieux les francophones du Canada, on comprend qu'il n'est pas déraisonnable pour cette minorité de vouloir préserver leur langue et leurs institutions qui sont menacées par la simple prépondérance de la majorité.

Après une brève notice biographique de De Gaspé, Roberts explique que Les Anciens Canadiens était accompagné de notes et clarifications. Pour lui, cette section est une masse d'informations curieuses qu'il ne traduit pas, car elle revêt peu d'intérêt pour le lecteur moyen (6). Laissez-nous plutôt supposer que Roberts n'avait ni le temps, ni le goût, de traduire la centaine de pages que constituent les notes et clarifications écrites par De Gaspé et placées en fin d'ouvrage. Rappelons qu'il accepte de traduire le roman en moins d'un été, car il a sérieusement besoin d'argent. Il s'agit ici à notre avis d'une contradiction importante. Dans sa préface, Roberts souligne l'importance pour les anglophones d'en apprendre davantage sur les Canadiens-français, mais affirme vers la fin qu'une centaine de pages de notes portant explicitement sur les particularités de la vie et de l'histoire des Canadiensfrançais n'est pas d'une grande importance. Si son but au moment de réaliser la traduction est vraiment de faciliter la compréhension des deux peuples, il est contradictoire d'affirmer que la fiction a revêt un plus grand intérêt que les notes explicatives, et qu'ainsi cela justifie leur non traduction. Au final, nous en venons à la conclusion que Roberts se sent probablement obligé de justifier son omission, mais son argumentation tombe à plat.

Pour terminer, Roberts vante le style charmant, vieillot et peu pressé de l'auteur. La trame narrative du roman, bien que directe, se permet des diglossies pour introduire des représentations festives et des chansons. Dans ses dernières lignes, Roberts s'attarde sur Les Mémoires, ouvrage qui traite de la vie des seigneurs et des habitants du temps d'Aubert de Gaspé. 
En 1905, la traduction de Roberts est réimprimée sous le titre Cameron of Lochiel. Cette édition reprend la préface de 1890, mais la précède d'une "preface to the new edition » qui est également signé de la main de Roberts. Dans ce court texte de moins d'une page et demie, Roberts met de l'avant le fait que, selon lui, Cameron of Lochiel est le véritable héros de l'histoire de De Gaspé. Il justifie également le nouveau titre en disant que cela permet de conférer à l'auteur le titre/statut d'auteur de fiction qui lui revient.

Pour la première fois, il explique explicitement ce qui l'a poussé à produire une version anglaise. Il affirme que ce n'est pas la qualité littéraire des Anciens Canadiens qui l'intéressait, mais bien le fait que la trame narrative regorge de descriptions des traditions, du folklore et des coutumes de Canadiens-français. Il souligne également qu'il souhaitait contribuer, bien que ce soit d'une manière très modeste, à la compréhension mutuelle des deux Canadas : « ... my own anxiety to contribute, in however humble way, to the increase of understanding and confidence between the two great branches of the Canadian people » (De Gaspé 1905 ii). D'ailleurs, à son avis, l'œuvre d'Aubert De Gaspé met de l'avant des personnages aux grands idéaux et qu'à la lecture, cette histoire permet de bien comprendre les francophones.

Nous voyons ici que Roberts reprend, quoique plus brièvement, le thème des « deux solitudes » et de la nécessité pour les locuteurs de deux langues-cultures d'apprendre à mieux se connaître et se comprendre. Roberts est un nationaliste canadien-anglais vivant dans les Maritimes. Au Nouveau-Brunswick, il côtoie donc probablement des Acadiens, ce qui a pu le rendre plus sensible aux enjeux linguistiques des francophones. D'ailleurs, les revendications des Québécois ne le laissent pas indifférent. En 1890, il écrit qu'il est normal que les Québécois cherchent à sauvegarder leur langue et leurs institutions. À son avis, la traduction de la littérature des deux Canadas est donc un moyen de construire des ponts entre les deux nations. Il affirme donc que c'est dans cette optique qu'il a choisi de traduire Les Anciens Canadiens. Traduire un ouvrage phare du Canada français dans le but d'augmenter la compréhension qu'ont les anglophones de cette nation est un objectif fort louable. Toutefois, nous savons bien que pour Roberts l'incitatif premier de la traduction est le montant de deux cents dollars que lui a offert Appleton. Toutefois, nous croyons que Roberts, en tant que nationaliste canadien, est fort conscient du fossé qui sépare les Canadiens-anglais des Canadiens-français. Ainsi, il croit vraiment que les deux peuples doivent apprendre à mieux se connaître et que la traduction littéraire est un moyen d'y parvenir.

Nous aimerions maintenant nous pencher sur la deuxième retraduction, qui paraît en 1996 et est signée par Jane Brierley. La traductrice a précédemment traduit les deux autres ouvrages d'Aubert de Gaspé : Mémoires (A Man of Sentiment: The Memoirs of Philippe-Joseph Aubert de Gaspé) en 1988 et Divers (Yellow-Wolf \& Other Tales of the Saint Lawrence) en 1990. Jane Brierley est une traductrice littéraire reconnue : sa traduction Yellow-Wolf \& Other Tales of the Saint Lawrence est lauréate du Prix du Gouverneur général. Quatre autres de ses traductions ont également été mises en nomination pour ce prix, dont sa traduction des Anciens 
Canadiens. Elle a également été présidente de l'ATTLC (Association des traducteurs et traductrices littéraires du Canada ${ }^{2}$.

Jane Brierley appose ses initiales à une introduction de huit pages. Dans un premier temps, elle présente l'œuvre originale et son auteur. Pendant plus de deux pages, elle relate la vie et la carrière d'Aubert de Gaspé. Bien que ces informations puissent être intéressantes pour le lecteur anglophone, nous ne nous y attarderons pas, car il s'agit d'une présentation factuelle. Brierley passe ensuite aux Anciens Canadiens en tant qu'œuvre. Elle mentionne qu'il s'agit du premier roman à mettre en scène des personnages, des évènements et des valeurs d'un monde qui n'est plus (De Gaspé 1996: 11). Nous revoyons ici le thème de la mémoire également présent chez Roberts : Les Anciens Canadiens est un ouvrage important, car il couche sur papier une époque révolue de l'histoire canadienne, à savoir les mœurs des anciens colons français.

Tout comme Roberts, Brierley vante les mérites du roman d'Aubert de Gaspé. Elle met toutefois l'auteur à l'avant-plan. Les Anciens Canadiens est un ouvrage exceptionnel, car De Gaspé est un homme cultivé et que la lecture et l'érudition ont toujours fait partie de sa vie (De Gaspé 1996: 12-13). Il faut toutefois mentionner que Jane Brierley produit sa traduction à la toute fin du $\mathrm{XX}^{\mathrm{e}}$ siècle, quelque cent années après celle de Roberts. En ce sens, une mise en contexte plus détaillée est de mise. Aujourd'hui, Philippe Aubert de Gaspé est aussi célèbre que son œuvre. Toutefois, il en demeure que pour les Québécois, Aubert de Gaspé n'est un nom familier que si l'on a des connaissances en histoire de la littérature québécoise. Ainsi, il n'est pas erroné de penser que bien des Canadiens anglophones n'ont jamais entendu parler de cet homme.

Ensuite, Brierley établit un parallèle entre Aubert de Gaspé et Sir Walter Scott. Elle postule que De Gaspé a été influencé par la production littéraire de Scott. Elle met de l'avant les liens entre la vie personnelle de deux hommes et démontre que De Gaspé a repris plusieurs des stratégies littéraires de Scott telles que l'inclusion de poésie et de chansons ainsi que le recours aux notes de bas de page et de fin de chapitre (pour de plus amples informations sur les influences littéraires de Scott sur Aubert de Gaspé, voir les travaux de Rainier Grutman). À notre avis, Brierley s'attarde sur cette influence littéraire afin de donner de la crédibilité au roman d'Aubert de Gaspé. L'auteur canadien-français peut ainsi être placé dans la même catégorie que le romancier écossais de renom.

Représentante de notre époque, Brierley s'attarde ensuite à la place des femmes dans le roman d'Aubert de Gaspé. Elle affirme que les personnages féminins que De Gaspé met en scène sont plus complexes qu'il n'y paraît à première vue. Les femmes de De Gaspé incarnent la force de caractère, la résilience et le sacrifice de soi. Pourquoi Brierley consacre-t-elle un paragraphe à décrire et à vanter les personnages féminins de l'œuvre qui, avouons-le, sont assez secondaires dans l'histoire? Il s'agit peut-être d'un clin d'œil aux articles publiés qui analysent justement la représentation des femmes dans Les Anciens Canadiens. Comme pour les remarques sur Scott, il nous semble que Brierley cherche à souligner et à se

\footnotetext{
${ }^{2}$ Ces informations proviennent de la notice bio-bibliographique disponible sur le site Internet de l'ATTLC. <http://www.attlc-ltac.org/bak/Brierley.htm>.
} 
positionner par rapport aux études et réflexions contemporaines sur Aubert de Gaspé et son œuvre. Il nous apparaît également que le fait de mentionner la représentation des femmes est une manière d'interpeller son lectorat féminin, une préoccupation qui était absente au XIX ${ }^{\mathrm{e}}$ siècle.

Finalement, contrairement à Roberts, Jane Brierley explique clairement pourquoi elle a choisi de retraduire Les Anciens Canadiens : «Why, since this novel has twice been translated into English, was it worthwhile preparing a new version? » (De Gaspé 1996: 15) Elle nous mentionne qu'elle a précédemment signé un article où elle examine les traductions de Pennée et Roberts ${ }^{3}$, article qu'elle résume dans la préface. À son avis, la version produite par Pennée, bien qu'assez juste, est alourdie par une prose verbeuse et maladroite, et sa syntaxe truffée de virgules confère une aura archaïque au texte. De son côté, la traduction de Roberts est beaucoup plus lisible. Selon Brierley, toutefois, il a privilégié une approche adaptative et est allé trop loin. Elle lui concède toutefois une certaine indulgence en raison du fait qu'il a dû produire sa traduction en un été. Cependant, il n'a pas rendu en anglais les notes et clarifications en plus d'avoir omis plusieurs sections de l'original sans justification. Pour Brierley, l'attitude de Roberts face aux Anciens Canadiens est hautaine : "His was a rather cavalier approach - designed, one can't help thinking, to give de Gaspé a fin-de-siècle facelift. » Bref, pour elle, en privilégiant l'idiomaticité, Roberts produit une version anglaise des Anciens Canadiens qui tend beaucoup trop vers l'acceptabilité et délaisse l'adéquation et le respect de la lettre (au sens bermanien) de l'original. Ainsi, Brierley propose sa traduction dans le but que les lecteurs, tant les nouveaux venus que ceux déjà familiers avec les Anciens Canadiens, puissent «make a fresh acquaintance with de Gaspé's delightful work. » Bref, Brierley nous mentionne ce qui selon elle cloche dans les traductions précédentes, mais elle n'explique pas son approche traductive. Elle ne donne aucun indice sur la manière dont elle a entrepris son projet de traduction. En fait, ces informations se trouvent plutôt dans un article savant publié l'année précédant la publication de sa traduction, en 1995.

Après avoir analysé les préfaces rédigées par Charles G. D. Roberts (1890, 1905) et Jane Brierley (1996), nous remarquons que les deux traducteurs mettent de l'avant des préoccupations bien différentes. Ainsi, la préface de Brierley est plutôt une introduction à la vie et à l'œuvre de Philippe Aubert de Gaspé. Écrite pour le lecteur canadien-anglais du tournant du $\mathrm{XXI}^{\mathrm{e}}$, sa préface privilégie les informations factuelles sur la vie d'Aubert de Gaspé et ce qui l'a amené à l'écriture. Elle se penche également sur l'influence qu'ont eue les œuvres de Walter Scott sur la production littéraire d'Aubert de Gaspé. Dans la préface de Brierley, c'est l'importance littéraire des Anciens Canadiens et d'Aubert de Gaspé qui est mise de l'avant. Chez Roberts, la traduction des Anciens Canadiens est plutôt justifiée par la nécessité pour les deux Canadas d'apprendre à mieux se connaître. La traduction de 1890 de Canadians of Old est donc un outil pour rapprocher les « deux

\footnotetext{
${ }^{3}$ Voir Brierley, Jane. « Two-and-a-half Translators in Search of a Canadian of Old». Culture in Transit : Translating the Literature of Quebec. Dir. Sherry Simon. Montréal: Vehicule Press, 1995.
} 
solitudes ». Roberts et Brierley n'ont en commun qu'un seul aspect : ils affirment tous les deux qu'il est crucial de traduire Les Anciens Canadiens, car ce roman a permis de préserver les mœurs des colons français de l'ancien régime. Ainsi, le seul thème à traverser les époques est celui de la mémoire. Aubert de Gaspé écrit Les Anciens Canadiens dans le but de préserver les mœurs et les coutumes d'une époque révolue et c'est pour cette même raison qu'il a été traduit et retraduit. 


\section{Bibliographie}

Arès, Richard. Notre question nationale, Volume 3. Éditions de l'Action nationale, 1945.

Aubert de Gaspé, Philippe (père). Les Anciens Canadiens. Québec : Desbarats et Derbishire, 1863.

---. The Canadians of Old. Trad. Georgiana M. Pennée. Québec : G. et G.E. Desbarats, 1864.

Aubert de Gaspé, Philippe. Canadians of Old. Trad. Charles G. D. Roberts. New York : D. Appleton and Company, 1890.

---. Cameron of Lochiel. Trad. Charles G. D. Roberts. Boston : L. C. Page \& Company, 1905.

---. Canadians of Old. A romance. Trad. Jane Brierley. Montréal : Véhicule Press, 1996.

« Brierley, Jane ». attlc-ltac.org. < http://www.attlc-ltac.org/bak/Brierley.htm $>$. n.d.

Brierley, Jane. « Two-and-a-half Translators in Search of a Canadian of Old». Culture in Transit: Translating the Literature of Quebec. Dir. Sherry Simon. Montréal : Vehicule Press, 1995.

Biron, Michel, François Dumont et Élisabeth Nardout-Lafarge. Histoire de la littérature québécoise. Montréal : Boréal, 2007.

Boone, Laurel. The Collected Letters of Sir Charles G.D. Roberts. Fredericton : Goose Lane Editions, 1989.

Lareau, Edmond. Histoire de la littérature canadienne. Montréal : John Lovell, 1874.

Linteau, Paul-André, René Durocher et Jean-Claude Robert. Histoire du Québec contemporain. Montréal : Boréal express, 1979. 